\title{
Prognostic impact of macrometastasis linear size in sentinel node biopsy for breast carcinoma
}

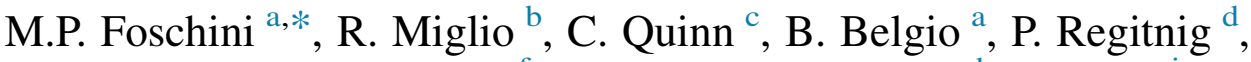 \\ S. Bianchi ${ }^{\mathrm{e}}$, R. Nannini ${ }^{\mathrm{f}}$, H. Buerger ${ }^{\mathrm{g}}$, H. Kaya ${ }^{\mathrm{h}}$, I. Illyés ${ }^{\mathrm{i}}$, \\ J. Kulka ${ }^{i}$, C.A. Wells ${ }^{j}$, J. De Gaetano ${ }^{k}$, I. Lipeniece-Karele ${ }^{1}$, \\ G. Cserni ${ }^{\mathrm{m}, \mathrm{n}}$
${ }^{a}$ Department of Biomedical and Neuromotor Sciences, Section of Anatomic Pathology at Bellaria Hospital, University of Bologna, Bologna, Italy
${ }^{\mathrm{b}}$ Department of Statistical Sciences "Paolo Fortunati", University of Bologna, Bologna, Italy ${ }^{\mathrm{c}}$ St Vincent's University Hospital, Dublin, Ireland
${ }^{\mathrm{d}}$ Institute of Pathology, Medical University of Graz, Auenbruggerplatz 25, 8036 Graz, Austria Florence, Italy
${ }_{\mathrm{f}}^{\mathrm{f}}$ Division of Anatomic Pathology, District Hospital, Imola, Bologna, Italy
${ }^{\mathrm{g}}$ Institute of Pathology, Breast Cancer Center Paderborn, Husener Str.46a, 33098, Paderborn, Germany
${ }^{\mathrm{h}}$ School of Medicine, Department of Pathology, Marmara University, Istanbul, Turkey
${ }^{\mathrm{i}}$ 2nd Department of Pathology, Semmelweis University Budapest, Üllöiút 93, Budapest, 1091, Hungary
${ }^{\mathrm{j}}$ Department of Pathology, University College London, Level 2, Rockefeller Building, 21, University Street, London, WC1E 6JJ, UK
${ }^{\mathrm{k}}$ Department of Pathology, Mater Dei Hospital, Tal-Qroqq, Msida, MSD 2090, Malta
${ }^{1}$ Pathology Centre, Riga East Clinical University Hospital, Hipokrata St 2, Riga, LV-1038, Latvia
${ }^{\mathrm{m}}$ Bács-Kiskun County Teaching Hospital, Nyiriut 38, Kecskemét, 6000, Hungary \\ ${ }^{\mathrm{e}}$ Division of Pathological Anatomy, Department of, Surgery and Translational Medicine, University of Florence, \\ ${ }^{\mathrm{n}}$ Department of Pathology, University of Szeged, Allomás u. 1, Szeged, 6720, Hungary
}

Accepted 5 May 2017

Available online 17 May 2017

\begin{abstract}
Aim: The aim of the present study was to evaluate the risk of axillary non-sentinel lymph-node metastases (ALN) in breast cancer patients presenting macrometastasis (Mac-m) in the sentinel lymph node (SN).

Materials and methods: A retrospective series of 1464 breast cancers from patients who underwent ALN dissection following the diagnosis of Mac-m in the sentinel node (SN) was studied. In all the cases the MAC-m linear size was evaluated and correlated with presence or absence of non-SN ALN metastases.

Results: Non-SN metastases were detected in $644 \backslash 1464$ cases $(43.98 \%)$. The risk of further axillary metastases ranged from $20.2 \%$ (37/ 183 ) in cases with Mac-m between 2 and $2.9 \mathrm{~mm}$, to 65.3\% (262/401) in cases with Mac-m measuring > $10 \mathrm{~mm}$. The risk of non-SN ALN metastases showed a 3\% increase, parallel to each $\mathrm{mm}$ increment in SN metastasis size. The data evaluated with the receiver operating characteristic (ROC) curve showed that the Mac-m could be subdivided according to a new cut-off of $7 \mathrm{~mm}$. pT1 tumours, with Mac-m $<7 \mathrm{~mm}$ had a risk of non-SN ALN metastases of $<30 \%$. Furthermore 109/127 of these (85.8\%) had 3 or less non-SN ALN -metastases. Conclusions: The present data give a detailed description on the risk of non-SN ALN involvement, that may be useful in the evaluation of breast cancer patients. It is suggested that a Mac-m size of $<7 \mathrm{~mm}$ is related to a low residual axillary disease burden in breast cancer patients with small (pT1) tumours.
\end{abstract}

(C) 2017 Elsevier Ltd, BASO The Association for Cancer Surgery, and the European Society of Surgical Oncology. All rights reserved.

Keywords: Sentinel node; Macrometastasis; Breast cancer; Axillary dissection; Axillary metastasis; Staging

\footnotetext{
* Corresponding author. Anatomic Pathology Bellaria Hospital, Via Altura 3, 40139 Bologna, Italy. Fax: +390516225759.

E-mail addresses: mariapia.foschini@ausl.bologna.it, mariapia.foschini@unibo.it (M.P. Foschini).
} 


\section{Introduction}

In patients with breast cancer, the wide application of SN biopsy (SNB) has led to a reduction of side effects occurring after axillary lymph node dissection (ALND).

SNs free of metastatic deposits, can reliably predict the absence of further metastases in the axilla. ${ }^{6}$ It is similarly clear that the presence or absence of metastatic deposits in the $\mathrm{SN}(\mathrm{s})$ cannot be the only feature to be evaluated, to predict further axillary node (ALN) involvement. ${ }^{1,2}$

The size of the metastatic deposits has been one of the most important and most studied parameters in this respect. ${ }^{5-7}$ It has been evident that small volume metastatic deposits in the $\mathrm{SN}$ were related to a low risk of further axillary metastases. Therefore, the SN metastatic deposits were arbitrarily classified into three size categories: isolated tumour cells (ITC) when not greater than $0.2 \mathrm{~mm}$; micrometastasis (Mic-m) when between 0.2 and $2 \mathrm{~mm}$ and macrometastasis (Mac-m) when larger than $2 \mathrm{~mm}$. These definitions, with some refinements, are currently applied in SN evaluation. ${ }^{8}$

Despite the diagnostic problems and pitfalls, ${ }^{9-12}$ it is evident that small volume metastatic deposits correspond to a low risk of further axillary involvement. ${ }^{13}$ Accordingly, the presence of ITCs, even if related to a small increase in the risk of axillary metastases ${ }^{14}$ was not considered enough to perform ALDN, which at that time was limited to patients with Mic-m and Mac-m.

In 2011, Giuliano et al. ${ }^{15}$ demonstrated that selected patients with metastatic disease, including Mic-m and limited Mac-m, can safely avoid ALND, when breast conserving surgery is combined with whole breast irradiation (RT) and patients receive adjuvant systemic treatment (mostly chemotherapy, CHT) as well. The results of the American College of Surgeons Oncology Group (ACOSOG) Z0011 trial published by Giuliano et al., ${ }^{15}$ even if subject to criticisms, ${ }^{16}$ have significantly changed the practice of axillary treatment in breast cancer, and have initiated a large scale validation study, the POSNOC (POsitive Sentinel NOdeadjuvant therapy alone versus adjuvant therapy plus Clearance or axillary radiotherapy) trial which aims to overcome the criticized aspects of the ACOSOG Z0011 trial. ${ }^{17}$ Two other randomized trials also support the omission of ALND in cases of SN Mic-m. ${ }^{18,19}$ Therefore, recently published guidelines, accept that ALND can be avoided in patients with early stage breast cancer and Mic-m, when matching the selection criteria of the Z0011 trial. ${ }^{1,2}$

Avoiding ALND in appropriately selected patients is expected to harbour no or very limited tumour burden in the axilla and greatly reduces the risk of local complications, leading to a better quality of life for patients without increasing the risk of disease recurrence.

In daily practice, it is evident that even patients with Mac-m in the SN who undergo ALND, are frequently free of further metastatic deposits, and in retrospect, receive unnecessary surgery with the potential risk of local complications.
On the other side, the arbitrary cut-off size to separate Mic-m from Mac-m, currently established at $2 \mathrm{~mm}$ based on a historical work by Huvos and colleagues introducing the term Mic-m, ${ }^{20}$ has been proposed without being validated in large series.

Recently Illyes et al., ${ }^{21}$ proposed that the $2 \mathrm{~mm}$ cut-off, could be modified to $6 \mathrm{~mm}$, allowing the avoidance of completion ALND in a larger proportion of patients.

In addition, numerous papers demonstrated that the size of the metastatic deposit in the SN is not the only parameter to consider the risk evaluation of further axillary involvement. Indeed, size and grade of the primary tumour, multifocality, presence of lympho-vascular invasion (LVI), extra capsular extension (ECE), are important parameters predicting the risk of further axillary involvement. $3,4,22-24$

Therefore, the aim of the present study was to retrospectively evaluate a large multi-institutional series of MAC-m in the SN with patients who underwent ALND, and to correlate the presence of further lymph-node metastatic involvement with the Mac-m linear size and primary tumour features. The final goal of the study was to provide data useful to evaluate the real risk of further axillary involvement in each breast cancer patient, in order to better tailor therapeutic planning.

\section{Materials and methods}

Cases were collected retrospectively from 13 European centres, all of which are involved in the diagnosis and treatment of screen detected breast cancer patients. All centres, but one (Imola, Bologna), are associated with the European Working Group for Breast Screening Pathology (EWGBSP), and therefore follow similar diagnostic protocols. The Imola centre, due to its geographical location very close to Bologna, shares the same diagnostic protocols with the Bologna Bellaria centre, and consequently the two centres were considered together.

Cases were included when they met the following criteria: a) SNB was performed at primary surgery for staging purposes; b) at least one SN contained a Mac-m; c) ALND with the removal of at least 10 axillary lymph nodes was performed in addition to the SNB; d) data on the primary tumour were available; e) no neo-adjuvant chemoor hormonal therapy had been administered. According to the inclusion criteria above, cases without metastatic deposits, or with either Mic-m or ITC in the SN, as well as male breast cancers were excluded. The cases therefore represent a consecutive series meeting the inclusion criteria from each centre.

In each case, the following parameters were evaluated: 1) largest linear size of the Mac-m, in millimetres ( $\mathrm{mm})$; 2) the number of SN involved by MAC-m; 3) presence of extra capsular extension (ECE). In addition, the following parameters were evaluated in the primary tumour: histotype, grade, size, presence of LVI and multifocality. 
Most of the cases were from a dual tracer labelling method using both a radiocolloid (99mTc labelled albumin) and patent blue dye. Tracer administration was either intra/ peritumoral and/or periareolar. All blue and/or hot nodes were considered SNs, palpable removed cold and non-dye labelled LNs were considered as non-SNs removed during SN biopsy. Intra-operative evaluation (based on frozen section and $\backslash$ or imprint cytology) was performed in about half of the cases, but intraoperative decision to perform ALND was based on the presence of a macrometastasis larger than $2 \mathrm{~mm}$. A summary of the methods applied in the different centres is given in Table 1S (see Supplementary files). In general, the centres did not follow the Z0011 trial based recommendations for the omission of ALND for cases of macrometastatic SLNs at the time of the data collection.

The diagnosis of Mac-m was performed according to the criteria previously published by the European Working Group on Breast Screening Pathology (EWGBSP)., 10

Histological typing, grading and staging of the primary tumour were performed according to currently accepted criteria. $^{25-27}$

\section{Statistical analyses}

Univariate analysis was used to evaluate all available variables as individual risk factors for Mac-m SN. Distribution of continuous variables was analysed with the Wilcoxon test, and the Chi-squared test was used for categorical variables.

For multivariate analysis, the variables were included into a logistic regression model using a backward stepwise selection. Variables with a $\mathrm{p}$ value greater than 0.05 were excluded from the final predictive model.

The subsequent multivariate predictive model was then validated internally on a predefined set of data and then through an external validation. Internal validation was based on cases from the two centres providing the highest number of cases (centres A and B). External validation was performed on the remaining centres.

Discrimination power of the model was evaluated by the area under the receiver operating characteristic (ROC) curve and the calibration of the model by the Hosmer-Lemeshow goodness-of-fit test. A cut-off value of less than $30 \%$ risk of metastatic ALNs was considered as low-risk. This cut-off was considered on the basis of the Z0011 trial ${ }^{15,28}$ where $27 \%$ of the patients in the ALND arm showed further axillary metastatic deposits and therefore this was the anticipated nodal positivity rate in the arm where ALND was omitted.

Sensitivity and specificity were determined for various risk estimates. All statistical tests were two-sided. Statistical analyses were done with STATA (ver. 12.0).

\section{Ethical considerations}

The present study did not influence patient's treatment, as it was performed anonymously and retrospectively.
The study was approved by the Ethical Committee at AUSL of Bologna, Italy (protocol n. 15080).

\section{Results}

A total of 1464 cases met the inclusion criteria and were collected for the present study.

All the patients were female, aged from 26 to 91 (mean: 58.5 , median 58).

Tumour histotype was: ductal, no special type (NST) in 1094 cases $(74.7 \%)$; lobular in 178 cases $(12.2)$; special types in 192 cases $(13.1 \%)$. As the number of special type carcinomas was small, they were evaluated altogether.

Tumour size ranged from 0.2 to $16 \mathrm{~cm}$, approximately half of the cases $(760 / 1464,51.9 \%)$ were between 1.5 and $2.5 \mathrm{~cm}$ (mean $2.2 \mathrm{~cm}$; median $1.9 \mathrm{~cm}$ ).

The average number of SNs examined was 2 (median 2).

The total number of non-SN, ALN examined was on average 15.4 (median 14, range from 10 to 35 ).

Non-SN metastases were detected in 644 cases (44\%). The number of non-SNs with metastatic deposits ranged between 1 and 35 (median mean: 3.8); 436 of 644 cases $(67.6 \%)$ presented $\leq 3$ non-SN involved.

ALN metastases in relation to the Mac-m size:

Tables 1 and 2

The risk of further axillary metastases ranged from $20.2 \%(37 / 183)$ in cases with MAC-m between 2.1 and $2.9 \mathrm{~mm}$, to $65.3 \%(262 / 401)$ in cases with Mac-m measuring $>10 \mathrm{~mm}$.

The risk of having metastatic non-SN ALNs was studied using a binary logistic regression model estimated on the internal validation series, based on cases from centres A and $\mathrm{B}$.

The logarithm of Mac-m size in each centre's series, histological tumour size, multifocality of the primary tumour, age (in classes: $26-40,41-50,51-60,61-70$ and over 70 years, the last group representing the reference category), number of SN metastasis, total number of SNs had a $p$ -value of less than 0.05 and were included in the multivariate analysis after a backward selection procedure.

Table 1

Risk of Metastatic non-SN Depending on size of Mac-M in SN.

\begin{tabular}{lll}
\hline Mac-msize in mm & $\begin{array}{l}\text { Number of metastatic } \\
\text { non-SN cases/total } \\
\text { number of cases }\end{array}$ & $\begin{array}{l}\text { Risk of metastatic } \\
\text { non-SN }\end{array}$ \\
\hline $2.1-2.9$ & $37 / 183$ & $20.2 \%$ \\
$3-3.9$ & $44 / 157$ & $28.1 \%$ \\
$4-4.9$ & $50 / 140$ & $35.7 \%$ \\
$5-5.9$ & $52 / 139$ & $37.4 \%$ \\
$6-6.9$ & $54 / 148$ & $36.5 \%$ \\
$7-7.9$ & $46 / 99$ & $46.5 \%$ \\
$8-8.9$ & $54 / 106$ & $50.9 \%$ \\
$9-9.9$ & $45 / 91$ & $49.5 \%$ \\
$\geq 10$ & $262 / 401$ & $65.3 \%$ \\
\hline
\end{tabular}


Table 2

Final predicted model using Mac-m size $7 \mathrm{~mm}$.

\begin{tabular}{|c|c|c|c|c|}
\hline & Coef. & $\mathrm{P}$-value & [95\% Conf. Interval] & Odds Ratio \\
\hline Mac-m $\geq 7$ & 0.91 & $<0.001$ & {$[0.62-1.21]$} & 2.50 \\
\hline $\begin{array}{l}\text { Tumour focality } \\
\text { (Yes/No) }\end{array}$ & 0.25 & $<0.001$ & {$[0.13-0.37]$} & 1.28 \\
\hline Age $26-40$ & -0.34 & 0.051 & {$[-0.68-0.00]$} & 0.71 \\
\hline Age $41-50$ & -0.35 & $<0.001$ & {$[-0.53-0.16]$} & 0.71 \\
\hline Age $51-60$ & -0.44 & $<0.001$ & {$[-0.57-0.31]$} & 0.65 \\
\hline Age $61-70$ & -0.5 & $<0.001$ & {$[-0.6-0.4]$} & 0.60 \\
\hline $\begin{array}{l}\text { Age } 71 \text { and over } \\
\text { [reference] }\end{array}$ & & & & 1.00 \\
\hline Tumour size & 0.03 & $<0.001$ & {$[0.02-0.05]$} & 1.03 \\
\hline $\begin{array}{c}\text { Number of SN } \\
\text { metastasis }\end{array}$ & 0.74 & 0.002 & {$[0.27-1.21]$} & 2.10 \\
\hline $\begin{array}{c}\text { Number of SN } \\
\text { examined }\end{array}$ & -0.4 & $<0.001$ & {$[-0.55-0.25]$} & 0.67 \\
\hline Constant & -1.56 & $<0.001$ & {$[-2.17-0.95]$} & \\
\hline
\end{tabular}

After this variable selection, we considered different integer cut-off values for Mac-m size and the value of $7 \mathrm{~mm}$ had the best discrimination power.

The final predicted model is reported in Table 3 using Mac-m size $7 \mathrm{~mm}$ or greater as explanatory variables along with the previously reported covariates.

The Hosmer-Lemeshow test produced a p-value of 0.538 indicating that the model fits and calibrates well for the patient population.

The ROC for the patient population used for estimating the model was 0.713 (95\% confidence interval: 0.680-0.746) suggesting a good discrimination.

The multivariate model predicted $694(74.30 \%)$ of the 934 patients in the original patient series to have metastatic non-SN ALN with a sensitivity of $85.58 \%$ and specificity of $35.03 \%$ at the $<30 \%$ cut-off threshold.

The predictive model was then validated by insertion of each patient's data from the external validation series into the equation of the binary logistic regression model. ROC for each internal and external validation centre are given in Table 3 .

Table 3

Performance of the predictive model in internal and external validation.

\begin{tabular}{llll}
\hline & $\mathrm{N}$ & $\begin{array}{l}\text { \% Mac-m } \\
\text { size } \geq 7 \mathrm{~mm}\end{array}$ & ROC \\
\hline Internal series & & & \\
Centres A + B & 934 & 51.39 & 0.713 \\
Centre A & 479 & 50.73 & 0.742 \\
Centre B & 455 & 52.09 & 0.681 \\
External validation & & & \\
C & 77 & 25.97 & 0.624 \\
D & 97 & 37.11 & 0.702 \\
E & 49 & 85.71 & 0.631 \\
F & 50 & 42.00 & 0.628 \\
F1 & 20 & 30 & 0.818 \\
F2 & 20 & 45 & 0.467 \\
F3 & 10 & 60 & 0.800 \\
G & 81 & 37.04 & 0.697 \\
H & 129 & 49.54 & 0.682 \\
\hline
\end{tabular}

An equation was deduced from the logistic regression analysis to predict a patient specific risk of having metastatic non-SNs, with p denoting the probability of this risk:

$$
\begin{aligned}
\operatorname{logit}(\mathrm{p})= & -1.56+0.91^{*} \mathrm{a}+0.25^{*} \mathrm{~b}-0.34^{*} \mathrm{c}-0.35^{*} \mathrm{~d} \\
& -0.44^{*} \mathrm{e}-0.5^{*} \mathrm{f}+0.03^{*} \mathrm{~g}+0.74^{*} \mathrm{~h}-0.4^{*} \mathrm{i}
\end{aligned}
$$

The letters in the equation denote the following variables: $\mathrm{a}=$ Mac-m $\geq 7 \mathrm{~mm}$ ( 0 if not present, 1 if present), $\mathrm{b}=$ tumour multifocality $(0$ if not present, 1 if present $)$, $\mathrm{c}=$ age $26-40(0$ if not present, 1 if present $), d=$ age $41-50(0$ if not present, 1 if present $), e=$ age $51-60$ (0 if not present, 1 if present $), \mathrm{f}=$ age $61-70(0$ if not present, 1 if present), $\mathrm{g}=$ tumour size $(\mathrm{mm}), \mathrm{h}=$ number of SNs with metastasis and $i=$ total number of SNs.

The predictive model is also provided as an excel-based calculator in the online only version of this article and at the website of European Working Group on Breast Screening Pathology (http://www.ewgbsp.org/) (Supplementary files).

\section{ALN metastases in relation to the number of $S N$ with} Mac-m: Table 4.

The number of metastatic SNs influenced the risk of further axillary node involvement, that ranged from $29.8 \%$ when $1 \mathrm{SN}$ only presented Mac-m, to $100 \%$ in the rare cases showing $>4$ metastatic $\mathrm{SN}$.

\section{ALN metastases in relation with Mac-m and tumour size: (Focus of $<7 \mathrm{~mm}$ ) (Table 5)}

Considering $7 \mathrm{~mm}$ as cut-off point and relating the presence of further axillary node metastases with the tumour size, it is evident that pT1 cases present a risk of further axillary metastatic involvement below 30\%. The risk increases to $38 \%$ in pT2 cases. On the contrary, when the SN Mac-m is larger than $7 \mathrm{~mm}$, the risk is higher than $50 \%$ in pT1c cases, reaching $84.6 \%$ in pT3 $\backslash$ pT 4 cases. In addition, the number of non-SN ALN greatly increased using $7 \mathrm{~mm}$ as cut off. Cases presenting as pT1a and pT1b had on average 1.59 metastatic non-SNs when the Mac-m was $<7 \mathrm{~mm}$, while the number of metastatic

Table 4

Non-sentinel node metastases in relation to the number of SN with Mac-m.

\begin{tabular}{ll}
\hline Number of Mac-M SN & $\begin{array}{l}\text { Number of metastatic non-SN } \\
\text { cases/total number of cases }\end{array}$ \\
\hline 1 & $186 / 624$ \\
& $29.8 \%$ \\
2 & $39 / 116$ \\
& $33.6 \%$ \\
3 & $9 / 24$ \\
4 or more & $37.5 \%$ \\
& $3 / 3$ \\
& $100 \%$
\end{tabular}

ALN: axillary lymph-node. 
Table 5

Relation between $\mathrm{pT}$ and further axillary metastatic involvement.

\begin{tabular}{lllll}
\hline SN Mac-m & pT1a + pT1b & pT1c & pT2 & pT3/pT4 \\
\hline$<7 \mathrm{~mm}$ & $19 / 88$ & $108 / 400$ & $99 / 258$ & $11 / 21$ \\
& $21.6 \%$ & $27 \%$ & $38.4 \%$ & $52.4 \%$ \\
Non-SN ALN & Range 1-3 & Range 1-12 & Range 1-13 & Range 1-13 \\
with MTS: & Average 1.59 & Average 2.3 & Average 2.48 & Average 4.18 \\
$\geq 7 \mathrm{~mm}$ & $26 / 45$ & $139 / 277$ & $220 / 349$ & $22 / 26$ \\
& $57 \%$ & $50.2 \%$ & $63.0 \%$ & $84.6 \%$ \\
Non-SN ALN & Range 1-21 & Range 1-28 & Range 1-35 & Range 1-22 \\
with MTS: & Average 5 & Average 3.84 & Average 4.70 & Average 8.68 \\
\hline
\end{tabular}

ALN almost doubled when the Mac-m size was $7.1 \mathrm{~mm}$ or more.

\section{ALN metastases in relation with Mac-m and tumour} grade: (Focus of $<7 \mathrm{~mm}$ )

Tumour grade alone seemed to be unrelated to the presence of further ALN metastases, as it ranged from $34.1 \%$ in G1 to $35.3 \%$ in G3. The mean numbers of ALNs involved by metastases were similar in G1 (2), G2 (2.2) and in G3 (2.5).

\section{ALN metastases in relation with Mac-m (Focus of $<7)$, grade and tumour size}

The importance of tumour grade emerged when associated with tumour size. Specifically, among G1 cases diagnosed at pT1a-pT1b, $15.8 \%$ only (3/19) presented further ALN metastases. On the contrary, the risk of further ALN metastases increased to $21.2 \%(11 / 52)$ in $\mathrm{G} 2$ and $29.4 \%$ $(5 / 17)$ in $\mathrm{G} 3$ cases.

In pT1c and pT2 cases, the risk of further ALN metastases is $37.8 \%$ (37/98) for G1 cases, $26.7 \%$ (87/326) for G2 and $35.5 \%(83 / 234)$.

$A L N$ metastases in relation with Mac-m, ECE, tumour multifocality and lymph-vascular invasion (pT3 and pT4 excluded) (Table 6, Supplementary files).

Tumour multifocality, diagnosed when more than one invasive carcinoma was detected, and LVI were confirmed to be important risk factors of further ALN metastatic

Table 6

Risk of further ALN metastases according to the presence of tumour multifocality, LVI and ECE.

\begin{tabular}{llllll}
\hline Mac-m & $<7 \mathrm{~mm}$ & & & All & \\
\cline { 2 - 3 } \cline { 6 - 6 } & Absent & Present & & Absent & Present \\
\hline Multifocality & $170 / 577$ & $56 / 169$ & & $435 / 1062$ & $176 / 355$ \\
& $29.5 \%$ & $33.1 \%$ & & $41.0 \%$ & $49.6 \%$ \\
LVI & $125 / 434$ & $101 / 312$ & & $281 / 748$ & $330 / 669$ \\
& $28.8 \%$ & $32.4 \%$ & & $37.6 \%$ & $49.3 \%$ \\
ECE & $124 / 479$ & $102 / 267$ & & $243 / 727$ & $368 / 690$ \\
& $25.9 \%$ & $38.2 \%$ & & $33.4 \%$ & $53.3 \% *$ \\
\hline
\end{tabular}

*P-value: $<0.0001$.
Table 7

ALN metastases in relation with age of the patient.

\begin{tabular}{ll}
\hline Age years & Further ALN metastases \\
\hline$\leq 40$ & $47 / 92$ \\
& $51.1 \%$ \\
$41-50$ & $125 / 303$ \\
& $41.3 \%$ \\
$51-60$ & $159 / 386$ \\
& $41.2 \%$ \\
$61-70$ & $149 / 375$ \\
& $39.7 \%$ \\
& $131 / 261$ \\
& $50.2 \%$ \\
\hline
\end{tabular}

$\mathrm{ALN}=$ axillary lymph-nodes.

involvement considering the cases altogether. On the contrary, tumour multifocality and LVI seemed to have a limited influence on the presence of non-SN ALN metastases in the Mac-m $<7 \mathrm{~mm}$ group.

The same phenomenon was observed considering ECE. This parameter had impact on further ALN metastases when the whole series was considered, while the impact was less evident when the group of SN Mac-m $<7 \mathrm{~mm}$ was considered.

ALN metastases in relation with Mac-m and age of the patient (pT3 and pT4 excluded) (Table 7 , Supplementary files):

Age influenced the presence or absence of further ALN metastases in the group of Mac-m $<7 \mathrm{~mm}$. Patients older than 70 or younger than 40 , had a higher risk of further ALN involvement.

A logistic regression model was estimated in the subgroups of patients with Mac-m $<7 \mathrm{~mm}$ and after a backward selection procedure the same variables were selected as relevant for predicting ALN involvements for the whole group (Table 8, supplementary files).

The ROC for the patient population, used for estimating the model $(\mathrm{n}=454)$ was $0.642(95 \%$ confidence interval: 0.586-0.698), suggested a discrete discrimination.

Table 8

Relevant data for predicting non-sentinel node involvements for the whole group

\begin{tabular}{lllll}
\hline & Coef. & P-value & {$[95 \%$ Conf. Interval] } & $\begin{array}{c}\text { Odds } \\
\text { Ratio }\end{array}$ \\
\hline $\begin{array}{l}\text { Tumour multifocality } \\
\quad \text { Yes/No) }\end{array}$ & 0.11 & 0.05 & {$[0.00-0.22]$} & 1.11 \\
Age 26-40 & & & & \\
Age 41-50 & -0.18 & 0.16 & {$[-0.50-0.14]$} & 0.84 \\
Age 51-60 & -0.3 & 0.5 & {$[-1.28-0.68]$} & 0.74 \\
Age 61-70 & -0.72 & 0.13 & {$[-0.97-0.47]$} & 0.49 \\
Age 71 and over & -0.88 & 0.14 & {$[-1.16-0.6]$} & 0.41 \\
$\quad$ reference] & & & & \\
$\begin{array}{l}\text { Tumour size } \\
\text { Number of SN metastasis }\end{array}$ & 0.03 & $<0.01$ & {$[0.02-0.03]$} & 1.03 \\
$\begin{array}{l}\text { Number of SN examined } \\
\text { Constant }\end{array}$ & -0.32 & 0.17 & {$[-0.07-0.95]$} & 1.66 \\
\hline
\end{tabular}




\section{Discussion}

The exact evaluation of the axillary metastatic burden is important as it may help to tailor therapy and avoid both over-and under-treatment in breast cancer patients. Several papers report on nomograms useful in the risk evaluation of further axillary metastatic involvement in the case of SN metastasis, ${ }^{7,8}$ but most of them are based on the wellknown classification into ITC, Mic-m and Mac-m.

It is now accepted that patients with ITC or Mic-m in the SN should not undergo ALND, ${ }^{1,2}$ especially when further radio and chemotherapy is planned.

Nevertheless, in daily practice, especially in centres working mainly with screen detected breast cancers, it is not unusual to detect cases with small Mac-m or encounter patients who receive mastectomy and do not need any further radiotherapy.

The presented data aim to improve correct therapeutic planning of these latter patient groups.

The data presented demonstrate that the presence of further axillary metastatic lymph-nodes is closely related to the SN Mac-m linear size and the characteristics of the primary breast tumour.

The data evaluated with the ROC curve showed that the Mac-m could be subdivided according at a cut-off of $7 \mathrm{~mm}$.

The linear size of $7 \mathrm{~mm}$, proposed here, is similar to that of $6 \mathrm{~mm}$ suggested in a previous study. Illyes et al. (21) measured the size of the SN metastasis in 75 SNs containing Mac-m. According to their results, the rate of axillary non-SN involvement was significantly higher in cases with Mac-m measuring 6,7 and $8 \mathrm{~mm}$. Therefore they suggested using Mac-m size of $6 \mathrm{~mm}$ as the cut-off value to mandate recommendation of ALND.

According to the results obtained here, three scenarios appeared.

The first and more favourable was that of pT1 tumours with Mac-m measuring less than $7 \mathrm{~mm}$. The second was that of pT3 $\backslash \mathrm{T} 4$ tumours with Mac-m larger than $7 \mathrm{~mm}$ reflecting a higher rate of non-SN involvement. As often happens in medicine, an intermediate group also appears, constituted by pT2 cases or by smaller tumours with Mac-m larger than $7 \mathrm{~mm}$.

Early stage breast cancer, especially the pT1 cases, with Mac-m smaller than $7 \mathrm{~mm}$ had a risk of further axillary metastatic involvement of $<30 \%$. In addition, the large majority of the cases $(109 / 127,85.8 \%)$ had 3 or less non-SN axillary metastatic lymph nodes.

Further features, as grade, multifocality, LVI and ECE had an impact on the risk evaluation, but were less relevant than Mac-m and tumour size.

These data are consistent with those presented in a previous study by the EWGBSP, based on small breast cancers, being less than $15 \mathrm{~mm}$. Cserni et al. ${ }^{29}$ studied 2929 breast tumours and related the type of SN metastatic deposit with further axillary involvement. According to their results, tumour and SN metastatic size were strongly correlated with non-SN involvement. Their rate of $38 \%$ of Mac-m showing further axillary involvement is consistent with the present 36\% (292/810) of pT1 cases showing further axillary metastases.

Subdividing Mac-m according to the herein proposed cut-off of $7 \mathrm{~mm}$, the risk of further axillary involvement dropped to $21.6 \%$ in pT1a $\backslash \mathrm{T} 1 \mathrm{~b}$ tumours and to $27 \%$ in pT1c tumours.

The rates of non-SN involvement reported in this series in $\mathrm{pT} 1 \mathrm{a} \backslash \mathrm{T} 1 \mathrm{~b}$ tumours are not so different from the risk observed in Mic-m, that ranges from 10 to $15 \%$ of the cases. $^{13,28}$

Cases presenting with pT2 tumours with Mac-m $<7 \mathrm{~mm}$, showed a 38\% risk of further axillary lymph node involvement. However, the TNM definition of pT2 includes a wide size range from $20.1-50 \mathrm{~mm}$. As the linear regression data showed, tumour size had a significant impact on the risk of further axillary involvement, the nomogram considered tumour size as a continuous variable in $\mathrm{mm}$, rather than the pT categories in order to more accurately classify the pT2 cases.

Cases presenting with a Mac-m $>7 \mathrm{~mm}$ and pT3-4 tumours were located at the opposite end of the spectrum, with a risk of further axillary involvement approaching $90 \%$. In addition, the number of metastatic non-SNs was higher (mean 8.68, range 1-22) in this subset.

Recently published guidelines ${ }^{1,2}$ recommend the avoidance of ALND in patients with 1 or 2 metastatic SNs. These guidelines also recommend caution in patients with "large or bulky metastatic axillary SN and $\backslash$ or those with gross extranodal extension of the tumour". In addition these recommendations state that patients with large tumours (as T3\T4) should not undergo SNB.

The present series included only a few pT3-T4 tumours, as cases were mainly obtained from institutions working with screen detected breast cancers. However, the data collected on locally advanced breast cancers further confirm the high burden of axillary metastatic involvement in these cancers. This is also reflected by the application of the newly proposed $7 \mathrm{~mm}$ cut-off in locally advanced cases where the risk of further axillary involvement remains high, ranging from $61.1 \%$ when the Mac-m is $<7 \mathrm{~mm}$ to $84.6 \%$ when the Mac-m is $\geq 7 \mathrm{~mm}$.

Measurements of Mac-m size were derived from formalin-fixed and paraffin-embedded sections, but it is likely that the limit of $7 \mathrm{~mm}$ can also be applied to frozen sections.

In conclusion, the present data indicate that a SN Mac-m size of $<7 \mathrm{~mm}$ is related to a low residual axillary disease burden in breast cancer patients with small (pT1) tumours and suggest that completion ALND may be avoided in this group.

\section{Conflict of interest statement}

No conflict of interest to be disclosed. 


\section{Acknowledgements}

The paper was financed with grants from the University of Bologna (Fundamentally Oriented Research, RFO). This study was partially (Gabor Cserni) funded by the National Research, Development and Innovation Office grant GINOP-2.3.2-15-2016-00020, Sources of funds had no influence on the manuscript preparation.

\section{Appendix A. Supplementary data}

Supplementary data related to this article can be found at http://dx.doi.org/10.1016/j.ejso.2017.05.007.

\section{References}

1. Lyman GH, Temin S, Edge SB, et al. American Society of Clinical Oncology Clinical Practice. Sentinel lymph node biopsy for patients with early-stage breast cancer: American Society of Clinical Oncology clinical practice guideline update. J Clin Oncol 2014 May 1;32(13): 1365-83.

2. Lyman GH, Somerfield MR, Bosserman LD, Perkins CL, Weaver DL, Giuliano AE. Sentinel lymph node biopsy for patients with early-stage breast cancer: American society of clinical Oncology clinical practice guideline update. J Clin Oncol 2016 Dec 12. JCO2016710947. [Epub ahead of print].

3. Meretoja TJ, Leidenius MH, Heikkilä PS, et al. International multicenter tool to predict the risk of nonsentinel node metastases in breast cancer. J Natl Cancer Inst 2012 Dec 19;104(24):1888-96.

4. Meretoja TJ, Audisio RA, Heikkilä PS. International multicenter tool to predict the risk of four or more tumor-positive axillary lymph nodes in breast cancer patients with sentinel node macrometastases. Breast Cancer Res Treat 2013;138(3):817-27.

5. Degnim AC, Griffith KA, Sabel MS, et al. Clinicopathologic features of metastasis in non-sentinel lymph nodes of breast carcinoma patients. Cancer 2003;98:2307-15.

6. Cserni G. Sentinel node biopsy and nodal staging. In: KahánZs Tot T, editor. Breast cancer, a heterogeneous disease entity. The very early stages. Dordrecht-Heidelberg-London-New York: Springer Science+ Business Media BV; 2011. p. 149-84.

7. Van la Parra RF, Peer PG, Ernst MF, Bosscha K. Meta-analysis of predictive factors for non-sentinel lymph node metastases in breast cancer patients with a positive SLN. Eur J Surg Oncol 2011;37(4):290-9.

8. Edge S, Byrd DR, Compton CC, et al. AJCC cancer staging manual. 7 th ed. Springer; 2010.

9. Cserni G, Bianchi S, Boecker W, et al. European Working Group for Breast Screening Pathology. Improving the reproducibility of diagnosing micrometastases and isolated tumor cells. Cancer 2005;103(2):358-67.

10. Cserni G. Evaluation of sentinel lymph nodes in breast cancer. Histopathology 2005;46(6):697-702.

11. Turner RR, Weaver DL, Cserni G, et al. Nodal stage classification for breast carcinoma: improving interobserver reproducibility through standardized histologic criteria and image-based training. J Clin Oncol 2008;26(2):258-63.
12. Cserni G, Amendoeira I, Bianchi S, et al. Distinction of isolated tumour cells and micrometastasis in lymph nodes of breast cancer patients according to the new Tumour Node Metastasis (TNM) definitions. Eur J Cancer 2011;47:887-94.

13. Van Deurzen CH, de Boer M, Monninkhof EM. Non-sentinel lymph node metastases associated with isolated breast cancer cells in the sentinel node. J Natl Cancer Inst 2008;100(22):1574-80.

14. De Boer M, van Deurzen CH, van Dijck, et al. Micrometastases or isolated tumor cells and the outcome of breast cancer. N Engl J Med 2009 Aug 13;361(7):653-63.

15. Giuliano AE, Hunt KK, Ballman KV, et al. Axillary dissection vs no axillary dissection in women with invasive breast cancer and sentinel node metastasis. JAMA 2011;305:569-75.

16. Gatzemeier W, Mann GB. Which sentinel-node positive breast cancer patients needs an axillary lymph-node dissection - ACOSOG Z0011 results and beyond. Breast 2013;22:211-6.

17. Goyal A, Dodwell D. POSNOC: a randomised trial looking at axillary treatment in women with one or two sentinel nodes with macrometastases. Clin Oncol (R Coll Radiol) 2015;27(12):692-5.

18. Solá M, Alberro JA, Fraile M, et al. Complete axillary lymph node dissection versus clinical follow-up in breast cancer patients with sentinel node micrometastasis: final results from the multicenter clinical trial AATRM 048/13/2000. Ann Surg Oncol 2013;20:120-7.

19. Galimberti V, Cole BF, Zurrida S. International Breast Cancer Study Group Trial 23-01 Investigators. Axillary dissection versus no axillary dissection in patients with sentinel-node micrometastases (IBCSG 23-01): a phase 3 randomised controlled trial. Lancet Oncol 2013; 14:297-305.

20. Huvos AG, Hutter RVP, Berg JW. Significance of axillary macrometastases and micrometastases in mammary cancer. Ann Surg 1971;173: 44-6.

21. Illyes I, Tokes AM, Kovacs A, et al. In breast cancer patients sentinel lymph node metastasis characteristics predict further axillary involvement. Virchows Arch 2014;465(1):15-24.

22. Barranger E, Coutant C, Flahault A, Delpech Y, Darai E, Uzan S. An axilla scoring system to predict non-sentinel lymph node status in breast cancer patients with sentinel lymph node involvement. Breast Cancer Res Treat 2005;91(2):113-9.

23. Van Zee KJ, Manasseh DM, Bevilacqua JL, et al. A nomogram for predicting the likelihood of additional nodal metastases in breast cancer patients with a positive sentinel node biopsy. Ann Surg Oncol 2003; 10:1140-51.

24. Nadeem RM, Gudur LD, Saidan ZA. An independent assessment of the 7 Nomograms for predicting the probability of additional axillary nodal metastases after positive sentinel lymph node biopsy in a cohort of British patients with breast cancer. Clin Breast Cancer 2014;14:272-9.

25. Elston CW, Ellis IO. Pathological prognostic factors in breast cancer. I. The value of histological grade in breast cancer: experience from a large study with long-term follow-up. Histopathology 1991;19(5):403-10.

26. Tavassoli FA, Eusebi V. Tumors of the breast. 4 ed. Washington, DC: American Registry of Pathology/AFIP; 2009.

27. Lakhani SR, Ellis IO, Schnitt SJ, Tan PH, van de Vijver MJ, editors. WHO classification of tumours of the breast WHO/IARC classification of tumours, vol. 4. 4th ed. Lyon.

28. Cserni G, Bianchi S, Vezzosi V. Sentinel lymph node biopsy in staging small (up to $15 \mathrm{~mm}$ ) breast carcinomas. Results from a European multi-institutional study. Pathol Oncol Res 2007;13(1):5-14.

29. Giuliano A. Reply to I.A. Voutsadakis et al and A. Goyal et al. J Clin Oncol 2014;32(34):3902-4. 